\title{
Melanoma Screening with Computer Aided Dermoscopy System Based on
} the ABCD Criteria

\author{
Heller Richard ${ }^{1}{ }^{*}$ and Guilan Shi ${ }^{2}$. \\ ${ }^{1}$ School of Medical Diagnostics and Translational Science, Old Dominion University, Norfolk, VA 23508, USA. \\ ${ }^{2}$ Frank Reidy Research Center for Bioelectrics, Old Dominion Unviersity, Norfolk, VA 23508, USA.
}

* Corresponding Author : Heller Richard, School of MedicalDiagnosticsand Translational Science, Old Dominion University, Norfolk, VA 23508, USA .E-mail: rheller@odu.edu

Received date: February 04, 2019;Accepted date : February 28, 2019; Published date: March 14, 2019.

Citation : Heller Richard,Guilan Shi, Melanoma Screening with Computer Aided Dermoscopy System Based on the ABCD Criteria. Journal of Dermatology and Dermatitis. Doi:10.31579/2578-8949/052

Copyright : @ 2019 Heller Richard. This is an open-access article distributed under the terms of The Creative Commons Attribution License, which permits unrestricted use, distribution, and reproduction in any medium, provided the original author and source are credited.

\begin{abstract}
Melanoma mortality rates are the highest amongst skin cancer patients. The 5-year survival rate is $92 \%$ if melanoma is diagnosed at an early stage and is localized. If melanoma has spread to other parts of the body, survival is only $13 \%$. Therefore, it is particularly important to develop approaches that can enhance early and accurate diagnosis of melanoma. Dermatoscopy has been widely used to identify early tumor growth. With the addition of computer technology, digital dermatoscopy systems are capable of accurately assessing skin surface features modeled along the $\mathrm{ABCD}$ criteria and have been used for clinical assessment of pigmented skin lesions. This paper introduces a noninvasive computerized dermoscopy system that considers lesions on the skin for diagnosis.
\end{abstract}

\section{Background}

Melanoma is a lethal form of skin cancer, which originates from melanocytes. In 2019, the American Cancer Society estimated that there will be 96,480 new cases of melanoma in the United States and 7,230 deaths from the disease [1]. The global cancer statistics [2] also show that the incidence and mortality rates of melanoma are rising. Overall survival rate of patients diagnosed with melanoma is dependent on multiple factors including the thickness of the primary melanoma, involvement of draining and presence of disease at distant sites. For very early-stage melanoma which is localized at the primary site, 5-year survival rate is $92 \%$. Survival for melanoma that has spread to nearby lymph nodes is $63 \%$. If melanoma has spread to other parts of the body, the survival rate is only $13 \%$ [3]. Therefore, early and accurate diagnosis of melanoma is particularly important.

Rules most clinicians use for melanoma detection are generally derived from the $\mathrm{ABCDE}$ criteria: Asymmetry, irregular Borders, Multiple colors, Diameter $>6 \mathrm{~mm}$, Evolving (with respect to size, shape, shades of color, surface features, or symptoms) and the sevenpoint checklist $[4,5]$. The ABCDE rule has not been specific enough for consistent and accurate diagnosis. The lack of a quantifiable measurement makes it difficult to diagnose a skin lesion with certainty. Although diameter is easy to measure, it is not easy to judge the asymmetry or irregularity of a skin lesion by just looking at it. Below, we summarize the diagnostic approach using dermoscopic diagnosis according to established classification rules for detection and monitoring patients with melanoma.

Visual examination of sub surface structures of the skin can be performed in a non-invasive manner through dermoscopy which uses using incident light and oil immersion. Dermatoscopy increases the diagnostic accuracy of cutaneous melanoma when performed by trained observers [6, 7]. The dermatoscopic $\mathrm{ABCD}$ rule for melanocytic lesions has been developed to facilitate the diagnosis of melanoma from the interpretation of readings from the dermatoscope [8-10].

Differentiating melanoma from benign melanocytic nevi is not straight forward especially in early stages.

Auctores Publishing - Volume1-041 www.auctoresonline.org Page - 01
Although the detection of melanoma using dermoscopy is higher than unaided observation based detection, dermoscopic techniques require specialized training in pattern analysis. The diagnostic accuracy for melanoma in clinics varies from 56 to $80 \%$, according to experience and expertness of individual dermatologists [11]. Thus, an automatic diagnostic tool is essential for physicians. Computer aided evaluation is helpful to increase the diagnostics accuracy as well as speed.

\section{Asymmetry}

One of the important indicators that a lesion is possibly malignant melanoma is the asymmetry of a pigmented skin lesions. In the dermatoscopic ABCDE rule for melanocytic lesions, asymmetry contributes substantially to the diagnosis of melanoma [12]. Diagnosis, dependent on human visual interpretations, is highly subjective and has limited reproducible. Therefore, an objective assessment is required for automated quantification of asymmetric distribution of skin lesions.

Most of the existing asymmetry descriptors are based on the analysis of either geometrical shape [13] or color morphology [14] of cutaneous lesions independently. In some techniques, the symmetry feature is calculated based on geometrical measurements of the whole lesion, e.g. symmetric distance and circularity [15]. Other studies, propose the circularity index, as a measure of irregular borders in dermoscopic images $[16,17]$. H. F. Lorentzen and his colleagues used latent class analysis to assess the sensitivity of axis (a) symmetry. They analyzed ratings from four experts in dermatoscopy pigmented lesions by latent class analysis (LCA). Based on LCA, the asymmetry of a single lesion was determined [12]. More recently, researchers separately calculated shape and color asymmetries and then fused these individual features in the classification step [18]. However, asymmetry in shape, color and structure of cutaneous lesions are caused by the same pathological factor. Moreover, asymmetry of skin lesions might be underestimated, because shape and color asymmetric distributions are minimized, respectively, by different symmetry axes. This drawback may finally reduce sensitivity, whereas the most important objective for melanoma diagnosis is to maximize the identification of malignant lesions. Therefore, a novel asymmetry assessment for cutaneous lesions should be presented to simultaneously quantify the shape and pigmentation distributions. 
Zhao Liu's group proposes a novel asymmetry analysis based on a newly developed pigmentation elevation model and the global point signatures (GPSs). Specifically, for the identification of melanin and haemoglobin, the pigmentation elevation model was first constructed by computer based analysis of dermoscopic images. This new method can simultaneously quantify the distribution of shape and pigmentation of cutaneous lesions. In addition, the proposed GPS-based asymmetry analysis enables working on dermoscopic images from different databases and has proved to be inherently robust to external imaging variations. These advantages suggested that the proposed method has a high potential for follow-up treatment $[19,20]$.

\section{Irregular Borders and Invasion Depth}

The border structure provides important information for accurate diagnosis, for example, the clinical feature asymmetry is calculated from the border and atypical pigment networks, globules, and bluewhite areas also critically rely on the accuracy of border detection [21].

Though numerous methods have previously been developed for border detection in pigmented skin lesion images; most of these dealt with clinical images [22]. Recently, the research has focused more on dermoscopic images [23-27]. These methods include histogram thresholding [28] global thresholding on optimized color channels followed by morphological operations and hybrid thresholding [29].

Measurement of melanoma depth of invasion in skin tissues is of great significance in grading the variety of skin disease and for appropriately planning a patient's treatment [22]. Hence, depth is an important factor to diagnose. Traditionally, measuring the tumor depth is based on the examination of histopathological slides by a pathologist under a microscope or utilizing computer aided measurement of melanoma depth of invasion in microscopic images which is time consuming and prone to intra and inter observer variability. In one paper, Navid Noroozi's research group proposed a non-invasive computerized dermoscopy system that considers the estimated depth of skin lesions for diagnosis. A 3-D skin lesion reconstruction technique using the estimated depth obtained from regular dermoscopic images is presented. At first, cornified layer is segmented based on entropy of the ridges detected in the image. Then, segmentation of cell nuclei is done using color features and active contour model. Their method gives better results in samples with various epidermis structures [30].

\section{Multiple colors}

Skin lesion color is an important feature for diagnosing malignant melanoma [31-34]. The color features examined, include the percent melanoma color and a novel color clustering ratio. The color clustering ratio is a natural extension of the percent melanoma color feature to quantify the grouping of skin lesion pixels with colors characteristic of melanoma and to associate the physiological interpretation of that grouping with skin lesion discrimination.

Automatic detection of colors in dermoscopy images [35-37] using a number of techniques has been previously evaluated. This included relative color histogram analysis technique to determine color characteristic of melanomas in clinical images [33, 38]. Relative color [39-41] has been proposed as a method to avoid color distortion in imaging processes because of varying film types and lighting, as well as a method of accounting for variations in normal skin color. The relative color technique equalizes color changes because of widely varying skin types. The relative color histogram techniques $[33,38$, $41,42]$ supply the foundation for the fuzzy-based histogram analysis technique presented in some research. The technique presented in R.J. Stanley's experiment using the relative color to determine benign and malignant regions, which may be applicable to any set of images of benign and malignant lesions [43]. The use of relative color [39] in which the background color is subtracted from the image color, has been proposed as a technique to avoid color distortion in the imaging process as well as a method of accounting for variations in normal skin color.
Experimental results appear to indicate that the melanoma color feature information is located in the interior of the lesion, excluding the centralmost region, which accounts for approximately $10 \%$.

\section{Conclusion}

Sensitivity and specificity data from clinical studies indicated that computer-based diagnosis of melanoma does not greatly differ from the diagnostic capability of an expert clinician, and is independent of the optical acquisition method employed to analyze the lesions [44, 45]. Although the efficiency of using a computerized dermoscopy system to diagnose various skin lesions has been demonstrated by experimental results [46], digital image analysis will probably not replace direct physician observation and evaluation.

\section{References}

1. <cancer-facts-and-figures-2018.pdf>

2. F. Bray, J. Ferlay, I. Soerjomataram, R.L. Siegel, L.A. Torre, et al (2018) Global cancer statistics 2018: GLOBOCAN estimates of incidence and mortality worldwide for 36 cancers in 185 countries, CA: a cancer journal for clinicians, 68 (394-424).

3. S. Bhatia, S.S. Tykodi, J.A. Thompson (2009) Treatment of metastatic melanoma: an overview, Oncology (Williston Park, N.Y.), 23 (488-496).

4. F. Nachbar, W. Stolz, T. Merkle, A.B. Cognetta, T. Vogt, et al (1994) The $\mathrm{ABCD}$ rule of dermatoscopy. High prospective value in the diagnosis of doubtful melanocytic skin lesions, Journal of the American Academy of Dermatology, 30 (551-559).

5. N.R. Abbasi, H.M. Shaw, D.S. Rigel, R.J. Friedman, W.H. McCarthy, et al (2004) Early diagnosis of cutaneous melanoma: revisiting the ABCD criteria, Jama, 292 (2771-2776).

6. H. Lorentzen, K. Weismann, C.S. Petersen, F.G. Larsen, L. Secher, et al(1999) Clinical and dermatoscopic diagnosis of malignant melanoma. Assessed by expert and non-expert groups, Acta dermatovenereologica, 79 (301-304).

7. M. Binder, M. Schwarz, A. Winkler, A. Steiner, A. Kaider,et al (1995) Epiluminescence microscopy. A useful tool for the diagnosis of pigmented skin lesions for formally trained dermatologists, Archives of dermatology, 131 (286-291).

8. T. Fikrle, K. (2006) Pizinger, Dermatoscopic differences between atypical melanocytic naevi and thin malignant melanomas, Melanoma research, 16 (45-50).

9. A. Blum, H.P. Soyer, C. Garbe, H. Kerl, G. Rassner, et al (2003) The dermoscopic classification of atypical melanocytic naevi (Clark naevi) is useful to discriminate benign from malignant melanocytic lesions, The British journal of dermatology, 149 1159-1164.

10. G. Argenziano, G. Fabbrocini, P. Carli, V. De Giorgi, E. Sammarco, M. Delfino, (1998) Epiluminescence microscopy for the diagnosis of doubtful melanocytic skin lesions. Comparison of the ABCD rule of dermatoscopy and a new 7-point checklist based on pattern analysis, Archives of dermatology, 134 (1563-1570).

11. C.A. Morton, R.M. Mackie, (1998) Clinical accuracy of the diagnosis of cutaneous malignant melanoma, The British journal of dermatology, 138 (283-287).

12. H.F. Lorentzen, K. Weismann, F.G. Larsen, (2001) Structural asymmetry as a dermatoscopic indicator of malignant melanoma--a latent class analysis of sensitivity and classification errors, Melanoma research, 11 (495-501).

13. W.V. Stoecker, W.W. Li, R.H. Moss, (1992) Automatic detection of asymmetry in skin tumors, Computerized medical imaging and graphics : the official journal of the Computerized Medical Imaging Society, 16 (191-197).

14. S. Seidenari, G. Pellacani, C. Grana,(2006) Asymmetry in dermoscopic melanocytic lesion images: a computer description based on colour distribution, Acta dermato-venereologica,86(123128).

15. J. Jaworek-Korjakowska, R. Tadeusiewicz, (2015) Determination of border irregularity in dermoscopic color images of pigmented skin lesions, Conference proceedings : Annual International Conference of the IEEE Engineering in Medicine and Biology Society. IEEE Engineering in Medicine and Biology Society. Annual Conference, 2015 (2015) (2665-2668). 
16. A. Bono, S. Tomatis, C. Bartoli, G. Tragni, G. Radaelli, et al (1999) The ABCD system of melanoma detection: a spectrophotometric analysis of the Asymmetry, Border, Color, and Dimension, Cancer, 85 (72-77).

17. <Comparison of color clustering algorithms for segmentation of dermatological images.pdf $>$.

18. P. Schmid-Saugeona, J. Guillodb, J.P. Thirana, (2003) Towards a computer-aided diagnosis system for pigmented skin lesions, Computerized medical imaging and graphics : the official journal of the Computerized Medical Imaging Society, 27 65-78.

19. Z. Liu, L. Smith, J. Sun, M. Smith, R. Warr, (2011) Biological indexes based reflectional asymmetry for classifying cutaneous lesions, Medical image computing and computer-assisted intervention : MICCAI ... International Conference on Medical Image Computing and Computer-Assisted Intervention, 14 124132.

20. Z. Liu, J. Sun, L. Smith, M. Smith, R. Warr, (2012) Distribution quantification on dermoscopy images for computer-assisted diagnosis of cutaneous melanomas, Medical \& biological engineering \& computing, 50 (503-513).

21. Q. Abbas, I. Fondon, M. Rashid, (2011) Unsupervised skin lesions border detection via two-dimensional image analysis, Computer methods and programs in biomedicine, 104 e1-15.

22. H. Xu, R. Berendt, N. Jha, M. Mandal, (2017) Automatic measurement of melanoma depth of invasion in skin histopathological images, Micron (Oxford, England : (1993), 97 ( 56-67).

23. M.E. Celebi, H.A. Kingravi, H. Iyatomi, Y.A. Aslandogan, W.V. Stoecker, et al (2008) Border detection in dermoscopy images using statistical region merging, Skin research and technology : official journal of International Society for Bioengineering and the Skin (ISBS) [and] International Society for Digital Imaging of Skin (ISDIS) [and] International Society for Skin Imaging (ISSI), 14 (347-353).

24. S. Jain, V. jagtap, N. Pise, (2015) Computer Aided Melanoma Skin Cancer Detection Using Image Processing, Procedia Computer Science, 48 (735-740).

25. M. Emre Celebi, Q. Wen, S. Hwang, H. Iyatomi, et al (2013) Lesion border detection in dermoscopy images using ensembles of thresholding methods, Skin research and technology : official journal of International Society for Bioengineering and the Skin (ISBS) [and] International Society for Digital Imaging of Skin (ISDIS) [and] International Society for Skin Imaging (ISSI), 19 e252-258.

26. P. Schmid, (1999) Segmentation of digitized dermatoscopic images by two-dimensional color clustering, IEEE transactions on medical imaging, 18(164-171).

27. M. Emre Celebi, Y. Alp Aslandogan, W.V. Stoecker, H. Iyatomi,et al (2007) Unsupervised border detection in dermoscopy images, Skin research and technology : official journal of International Society for Bioengineering and the Skin (ISBS) [and] International Society for Digital Imaging of Skin (ISDIS) [and] International Society for Skin Imaging (ISSI), 13 (454-462).

28. H. Iyatomi, H. Oka, M. Saito, A. Miyake, M. Kimoto et al (2006) 183Quantitative assessment of tumour extraction from dermoscopy images and evaluation of computer-based extraction methods for an automatic melanoma diagnostic system, Melanoma research, (16-190).

29. <Automatic segmentation of dermoscopy images using histogram thresholding on optimal color channels.pdf $>$.

30. N. Noroozi, A. Zakerolhosseini, (2015) Computerized measurement of melanocytic tumor depth in skin histopathological images, Micron (Oxford, England : (1993), 77 (44-56)

31. F. Ercal, A. Chawla, W.V. Stoecker, H.C. Lee, R.H. Moss, (1994) Neural network diagnosis of malignant melanoma from color images, IEEE transactions on bio-medical engineering, 41 837-845.

32. $\mathrm{M}$

32. Auctores Publishing - Volume1-041 www.auctoresonline.org Page - 03
Landau, H. Matz, E. Tur, M. Dvir, S. Brenner, (1999) Computerized system to enhance the clinical diagnosis of pigmented cutaneous malignancies, International journal of dermatology, 38 443-446.

33. Y. Faziloglu, R.J. Stanley, R.H. Moss, W. Van Stoecker, R.P. McLean, (2003) Colour histogram analysis for melanoma discrimination in clinical images, Skin research and technology : official journal of International Society for Bioengineering and the Skin (ISBS) [and] International Society for Digital Imaging of Skin (ISDIS) [and] International Society for Skin Imaging (ISSI), 9 (147156).

34. H. Ganster, A. Pinz, R. Rohrer, E. Wildling, M. Binder, et al (2001) Automated melanoma recognition, IEEE transactions on medical imaging, 20 (233-239).

35. M. Elbaum, A.W. Kopf, H.S. Rabinovitz, R.G. Langley, H. Kamino, et al (2001) Automatic differentiation of melanoma from melanocytic nevi with multispectral digital dermoscopy: a feasibility study, Journal of the American Academy of Dermatology, 44 (207-218).

36. P. Rubegni, G. Cevenini, M. Burroni, G. Dell'Eva, P. Sbano, et al (2002) Digital dermoscopy analysis of atypical pigmented skin lesions: a stepwise logistic discriminant analysis approach, Skin research and technology : official journal of International Society for Bioengineering and the Skin (ISBS) [and] International Society for Digital Imaging of Skin (ISDIS) [and] International Society for Skin Imaging (ISSI), 8 (276-281).

37. M. Moncrieff, S. Cotton, E. Claridge, P. Hall, (2002) Spectrophotometric intracutaneous analysis: a new technique for imaging pigmented skin lesions, The British journal of dermatology, $146(448-457)$.

38. J. Chen, R.J. Stanley, R.H. Moss, W. Van Stoecker, (2003) Colour analysis of skin lesion regions for melanoma discrimination in clinical images, Skin research and technology : official journal of International Society for Bioengineering and the Skin (ISBS) [and] International Society for Digital Imaging of Skin (ISDIS) [and] International Society for Skin Imaging (ISSI), 9 (94-104).

39. S.E. Umbaugh, R.H. Moss, W.V. Stoecker, (1989) Automatic color segmentation of images with application to detection of variegated coloring in skin tumors, IEEE engineering in medicine and biology magazine : the quarterly magazine of the Engineering in Medicine \& Biology Society, 8 (43-50).

40. W.V. Stoecker, K. Gupta, R.J. Stanley, R.H. Moss, B. Shrestha, (2005) Detection of asymmetric blotches (asymmetric structureless areas) in dermoscopy images of malignant melanoma using relative color, Skin research and technology : official journal of International Society for Bioengineering and the Skin (ISBS) [and] International Society for Digital Imaging of Skin (ISDIS) [and] International Society for Skin Imaging (ISSI), 11 (179-184).

41. R.J. Stanley, W.V. Stoecker, R.H. Moss, (2007) A relative color approach to color discrimination for malignant melanoma detection in dermoscopy images, Skin research and technology : official journal of International Society for Bioengineering and the Skin (ISBS) [and] International Society for Digital Imaging of Skin (ISDIS) International Society for Skin Imaging (ISSI), 13 (62-72).

42. R.J. Stanley, R.H. Moss, W. Van Stoecker, C. Aggarwal, (2003) A fuzzy-based histogram analysis technique for skin lesion discrimination in dermatology clinical images, Computerized medical imaging and graphics: the official journal of the Computerized Medical Imaging Society, 27 (387-396).

43. R.J. Stanley, W.V. Stoecker, R.H. Moss, H.S. Rabinovitz, A.B. Cognetta, et al (2008) Basis function feature-based approach for skin lesion discrimination in dermatology dermoscopy images, Skin research and technology : official journal of International Society for Bioengineering and the Skin (ISBS) [and] International Society for Digital Imaging of Skin (ISDIS) [and] International Society for Skin Imaging (ISSI), 14 (425-435).

44. J. Mayer, (1997) Systematic review of the diagnostic accuracy of dermatoscopy in detecting malignant melanoma, The Medical journal of Australia, 167 (206-210). 
45. A. Blum, H. Luedtke, U. Ellwanger, R. Schwabe, G. Rassner, et al, (2004) Digital image analysis for diagnosis of cutaneous melanoma. Development of a highly effective computer algorithm based on analysis of 837 melanocytic lesions, The British journal of dermatology, 151 (1029-1038).

46.

T.Y. Satheesha, D. Satyanarayana, M.N.G. Prasad, K.D. Dhruve, (2017) Melanoma Is Skin Deep: A 3D Reconstruction Technique for Computerized Dermoscopic Skin Lesion Classification, IEEE journal of translational engineering in health and medicine, 54300117. 